\title{
Single-Pot Rapid Synthesis of Colloidal Core/Core-Shell Quantum Dots: A Novel Polymer-Nanocrystal Hybrid Material
}

\author{
Aarti Mehta $^{1}$, Shailesh N. Sharma ${ }^{1}$, Kanchan Sharma ${ }^{1,2}$, Parth Vashishtha ${ }^{1},{ }^{3}$ and S. Chand ${ }^{1}$ \\ ${ }^{1}$ National Physical Laboratory, Dr. K.S.Krishnan Road, New Delhi, 110012, India \\ ${ }^{2}$ Department of Physics, Delhi Technological University, New Delhi \\ ${ }^{3}$ Amity Institute of Nanotechnology, Amity University, Noida \\ E-mail: shailesh@mail.nplindia.ernet.in, shailesh@,nplindia.org
}

\begin{abstract}
Colloidal core and core shell Quantum Dots (QD's) are unique and important optoelectronic materials because properties of these QD's can be tailored by configuring core and optimizing shell thickness. In this research work, lead selenide (PbSe) core and PbSe-CdSe (Core-shell) QD's are synthesized using oleic acid as a capping ligand by colloidal route. This simpler, cost-effective and rapid single pot synthesis route for colloidal core-shell quantum dots unlike conventional double-pot approach like cationexchange and SILAR process has been reported for the very first time. Phase formation of prepared quantum dots is confirmed by XRD analysis, capping ligand presence by IR spectroscopy and morphological information by Scanning electron microscopy respectively. These synthesized inorganic quantum dots are dispersed in Poly (3-hexyl thiophene) polymer for formation of their respective nanocomposites. From PL quenching studies, it was inferred that $\mathrm{PbSe}-\mathrm{CdSe}$ coreshell quantum dots showed enhanced rate of PL quenching and hence higher value of Stern-Volmer constant $\left(K_{\text {sv }}\right)$ than PbSe Core QD's. This confirms that CdSe shell formation on PbSe core significantly passivates the core-surface, increases the stability and enhances the charge transfer mechanism for its potential application in Hybrid Solar cells.
\end{abstract}

Index Terms - Colloidal, PbSe core, PbSe-CdSe core-shell, Nanostructure, Hybrid

\section{INTRODUCTION}

IV-VI Colloidal semiconductors QDs are having their potential application in telecommunication, photoelectonic devices and biomedical labeling $[1,2]$.

These semiconductor QDs and their core shell structure can be synthesized by bottom up chemical synthesis. Shell formation of different semiconductor compound on the core structure is important to reduce the defect of core by passivating the electronic surface states of core and increase the stability of colloidal nanocrystal [3]. PbSe QDs are an important class of material having strong confinement effect due to large Bohr radius $(23 \mathrm{~nm})$ near about eight times larger than CdSe [4] and small band gap (0.28eV bulk) in near infrared region. It has been found that lead selenide QDs are not much stable and to stabilize the core different $\mathrm{PbSe} / \mathrm{PbS}$

] and $\mathrm{PbSe} / \mathrm{SiO}_{2}$ [6] core/shell structure are synthesized reported in literature. $\mathrm{PbSe} / \mathrm{CdSe}$ Core/Shell structures are also mentioned to improve the stability of core synthesized by SILAR and Cation exchanged process (double pot synthesis route).
In this research work, properties of $\mathrm{PbSe}$ core and $\mathrm{PbSe}$ CdSe core-shell QD's prepared by colloidal single pot synthesis route than conventional double pot synthesis routes like SILAR and Cation exchange process and polymer$\mathrm{PbSe} / \mathrm{PbSe}-\mathrm{CdSe}$ nanocomposites are reported for the first time. Cadmium selenide was opted as a shell material, due to its less lattice mismatch of $1 \%$ with $\mathrm{PbSe}$ [7] with marginal change of the surface chemistry and physics.

\section{EXPERIMENTAL DETAILS}

$\mathrm{PbSe}$ QDs are synthesized by chemical route method described by Murray et al [8]. Experiments are carried out under an Argon gas inert atmosphere using schlenk type apparatus.

\section{A. Required Chemicals}

Lead Acetate, Phenyl Ether, Oleic Acid, Oleylamine, Selenium metal powder and n-trioctyl phosphine (TOP) were purchased from Aldrich Chemical Co. P3HT poly (3-hexyl thiophene).

\section{B. Characterization}

XRD analysis has been performed for phase formation confirmation and crystallite size information by using Bruker D8 advance instrument. The morphology variations are confirmed by Scanning Electron microscopy and analysis is done by utilizing (SEM LS-440). Additionally, Fourier Transform Infrared spectroscopy was used for further confirmation of capping ligand and spectras of respective samples were recorded by Perkin Elmer Instrument (Spectrum BX-500).

\section{RESULTS \& DISCUSSIONS}

In this research work, $\mathrm{PbSe}$ core and $\mathrm{PbSe}-\mathrm{CdSe}$ core-shell (CS) QDs are synthesized by colloidal route using oleic acid as a capping ligand. These synthesized inorganic quantum dots (QDs) are dispersed in hexane for further characterization.

The presence of oleic acid capping ligand on the surface of $\mathrm{PbSe}$ core and $\mathrm{PbSe}-\mathrm{CdSe}$ core-shell QDs was confirmed by Fourier transform infrared spectroscopy. Fig.1 (a, b\&c) shows a stack view of IR spectra of Pure Oleic acid, PbSe Core and 
PbSe-CdSe core-shell (CS) QDs respectively. Two types of vibrations, mainly stretching and bending are responsible for most important peak and identification of different functional group.

In IR spectra of pure Oleic acid, the peak in the band (1650-1742) c. $\mathrm{m}^{-1}$ is the characteristic stretching vibration of $(\mathrm{C}=\mathrm{O})$ group present in carboxylic acid $(-\mathrm{COOH})$ and peak at $2911 \mathrm{c} . \mathrm{m}^{-1}$ is attributed due to the asymmetric $\mathrm{CH}_{2}$ stretching shown in fig. 1(a). Additionally the peaks in band 2880-2990 show the existence of $=\mathrm{C}-\mathrm{H}$ group and long alkyl chain present in the oleic acid [4]. The IR peaks in band 1100$1200 \mathrm{~cm}^{-1}$ confirms the presence of $\mathrm{P}=\mathrm{O}$ stretching mode corresponding to $(\mathrm{P}=\mathrm{O})$ in tri octyl phosphine (TOP) [9]. It can be clearly observed from fig.1 (b \& c), the peak obtained at $1734 \mathrm{~cm}^{-1}$ and $1730 \mathrm{~cm}^{-1}$ lies in the band (1650-1742) c.m confirms the presence of $(\mathrm{C}=\mathrm{O})$ due to oleic acid. The peaks at $1125 \mathrm{~cm}^{-1}$ and $1129 \mathrm{~cm}^{-1}$ are contributed by TOP ligand respectively. The presence of capping ligand on the surface of synthesized QDs reveals the efficient capping of oleic acid and TOP ligand on surface of lead selenide QDs and their core shell structure gives the evidence that they are strongly intact on the surface of nanocrystals used for surface passivation. It is well confirmed by IR spectroscopy that ligands are present on the surface and successfully capped on surface of respective synthesized QDs.

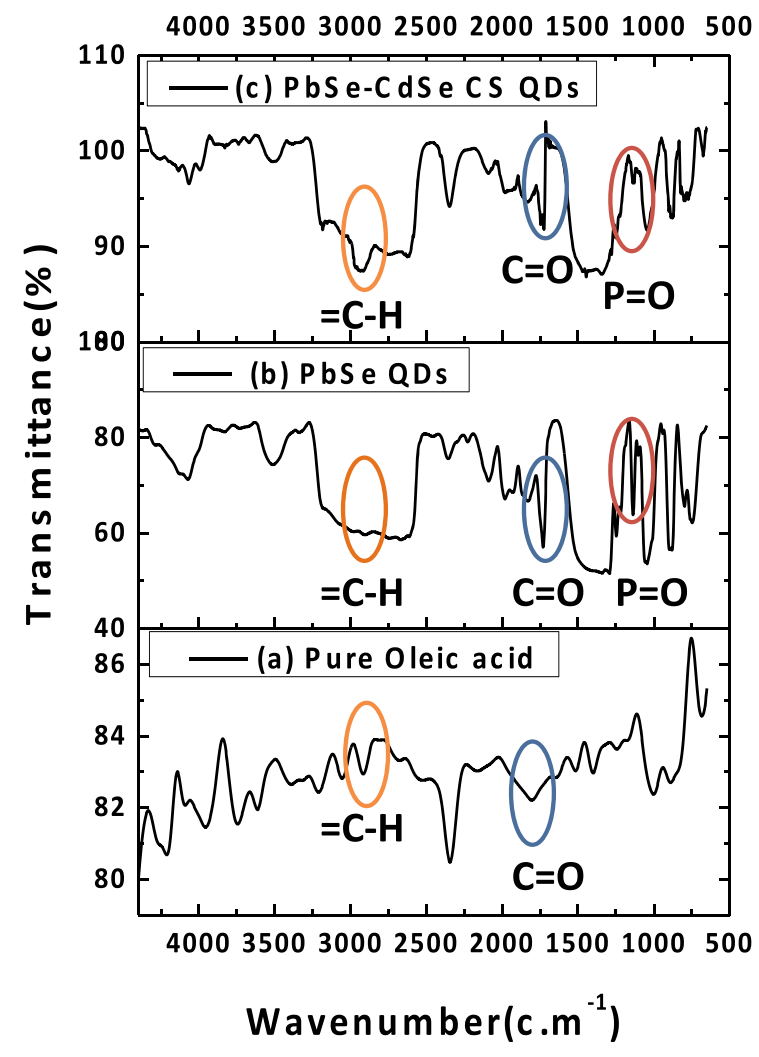

FIG.1. FTIR spectra of (a) Pure oleic acid (b) PbSe QDs (c) PbSeCdSe Core-shell QDs.
Fig. $2(\mathrm{a} \& \mathrm{~b})$ shows the XRD pattern of PbSe Core and $\mathrm{PbSe}-\mathrm{CdSe}$ core-shell structure respectively. The X-ray diffraction in fig. 2(a) exhibits clear peaks (111), (200), (220) matched with (JCPDS 78-1903) [10] data and confirms cubic rock salt crystal structure. It is clear from fig 1(b), the intensity (200) and (111) planes increased significantly and crystallinity is improved after shell formation due to effective passivation of core. XRD analysis gives the evidence that CdSe shell formation on PbSe core was successfully done. Morphology of these synthesized nanostructures was also confirmed by scanning electron microscopy.

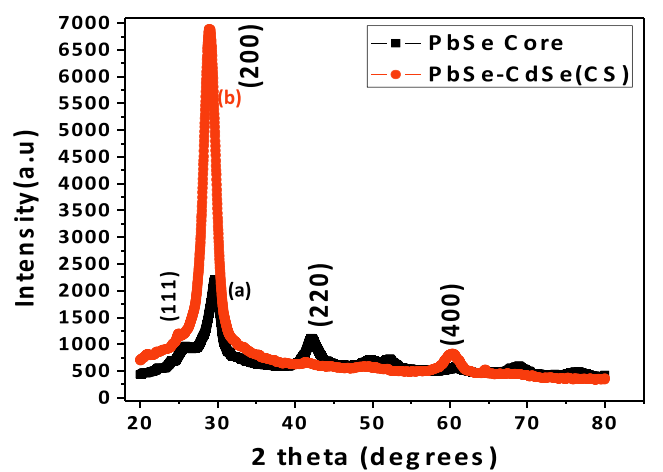

FIG.2. XRD spectra (a) PbSe Core (b) PbSe-CdSe Core-shell nanostructure.

Fig. $3(a, b)$ shows the SEM images for PbSe core and PbSe$\mathrm{CdSe}$ core-shell nanocrystallites respectively. It is clear from the figure 3. $(\mathrm{a}, \mathrm{b})$ that $\mathrm{PbSe}$ core and $\mathrm{PbSe}-\mathrm{CdSe}$ core shell structures are synthesized successfully by colloidal route. It might be perceived from inset of fig. 2(a) the synthesized $\mathrm{PbSe}$ core sample is spherical in shape and most of the part of morphology comes to be nonuniform in view of certain agglomeration effect expected in colloidal samples.

Fig.3 (b) shows the morphology of incorporated Pbse-CdSe core shell (CS) QDs and inset shows the structural data of same where two distinctive shade differentiations were watched the dark one speaks to the center part $(\mathrm{PbSe})$ and external partition of light grey color speaks to CdSe shell framing highlighted in figure. Morphological informations collected from SEM are also in well agreement with the results observed by XRD analysis of synthesized core and core shell nanostructures.

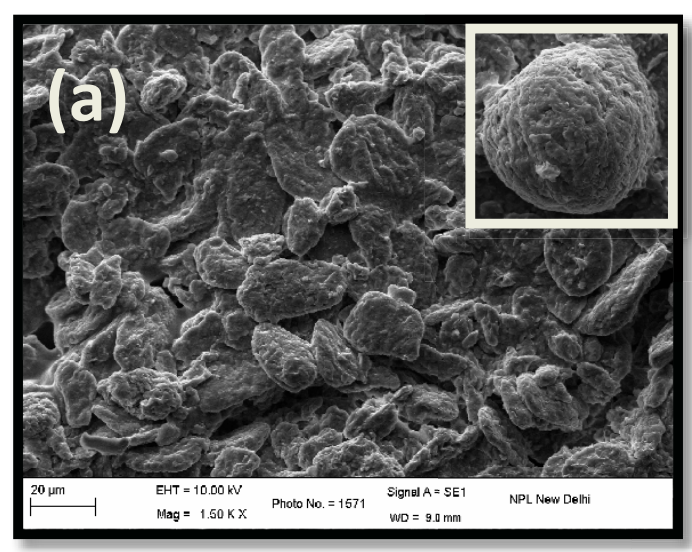




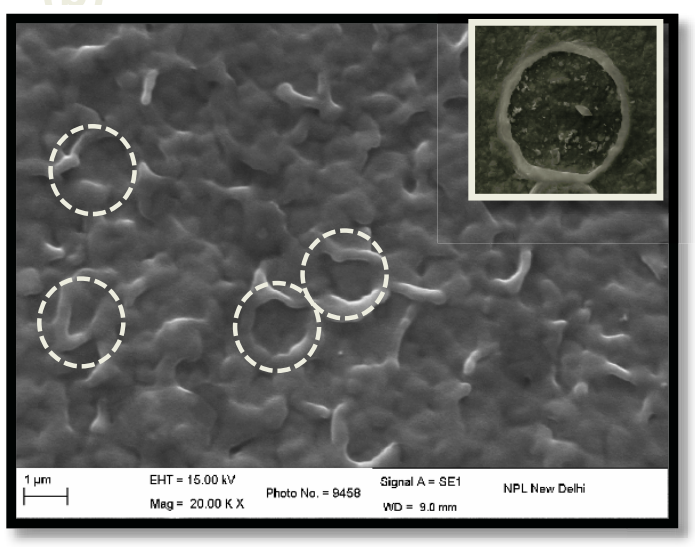

FIG.3. SEM images of (a) PbSe Core (b) PbSe-CdSe core-shell nanostructures.

After confirmation of phase formation and morphological analysis, photoluminescence (PL) Quenching experiment has been performed for further confirmation of charge transfer taking place from Polymer to inorganic QDs. For this, inorganic quantum dots are interacted with poly (3-hexyl thiophene) P3HT polymer matrix.

Basically there are two types of photoluminescence quenching: static and dynamic (collisional) [11, 12]. During quenching process fluorophore (polymer) interact with the quencher. In static quenching, complex is formed between polymer and quencher in ground state and it is basically nonfluorescent but in case of dynamic quenching the fluorophore interacts with the quencher during the life time of excited state and returns to the ground state without emission of a photon.

In case of collisional quenching; the following relation called the Stern-Volmer equation [11], holds:

$$
\mathrm{I}_{0} / \mathrm{I}=1+\mathrm{K}_{\mathrm{SV}}[\mathrm{Q}]
$$

Where, $\mathrm{I}_{0}$ and $\mathrm{I}$ are the fluorescence intensities observed in the absence and presence of quencher respectively, [Q] is the quencher concentration and $\mathrm{K}_{\mathrm{sv}}$ is the Stern-Volmer quenching constant. In a simplest case, a plot $\mathrm{I}_{0} / \mathrm{I}$ vs. [Q] should yield a straight line with a slope equal to $\mathrm{K}_{\mathrm{sv}}$ [11].

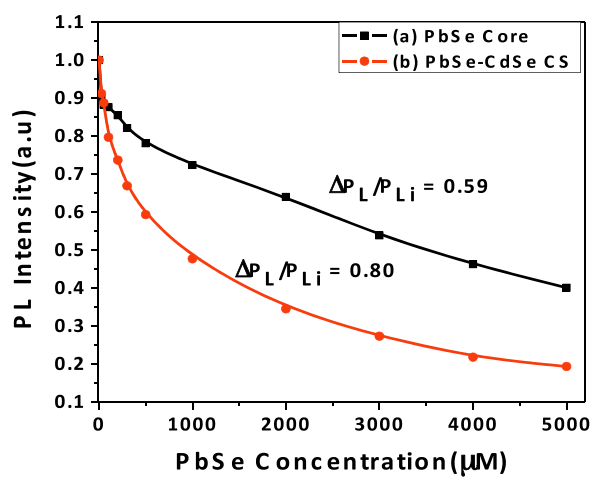

FIG.4. Variation of PL peak intensity of P3HT polymer with (a) $\mathrm{PbSe}$ core and (b) $\mathrm{PbSe}-\mathrm{CdSe}$ core-shell concentration.
Fig. 4 (a \& b) shows the emission intensity profiles of P3HT: $\mathrm{PbSe}$ (core and core-shell) nanocomposites as a function of concentration for both inorganic nanostructures. The rate of $\mathrm{PL}$ quenching $\left(\triangle \mathrm{P}_{\mathrm{L}} / \mathrm{P}_{\mathrm{Li}}\right)$ for $\mathrm{PbSe}$ core and $\mathrm{PbSe}-\mathrm{CdSe}(\mathrm{CS})$ QDs being (0.59) and (0.80) respectively.

Fig.5 (a \& b) shows the linear fitted Stern-Volmer plots for their respective nanocomposites plotted between $\mathrm{I}_{0} / \mathrm{I}$ versus QDs concentration.
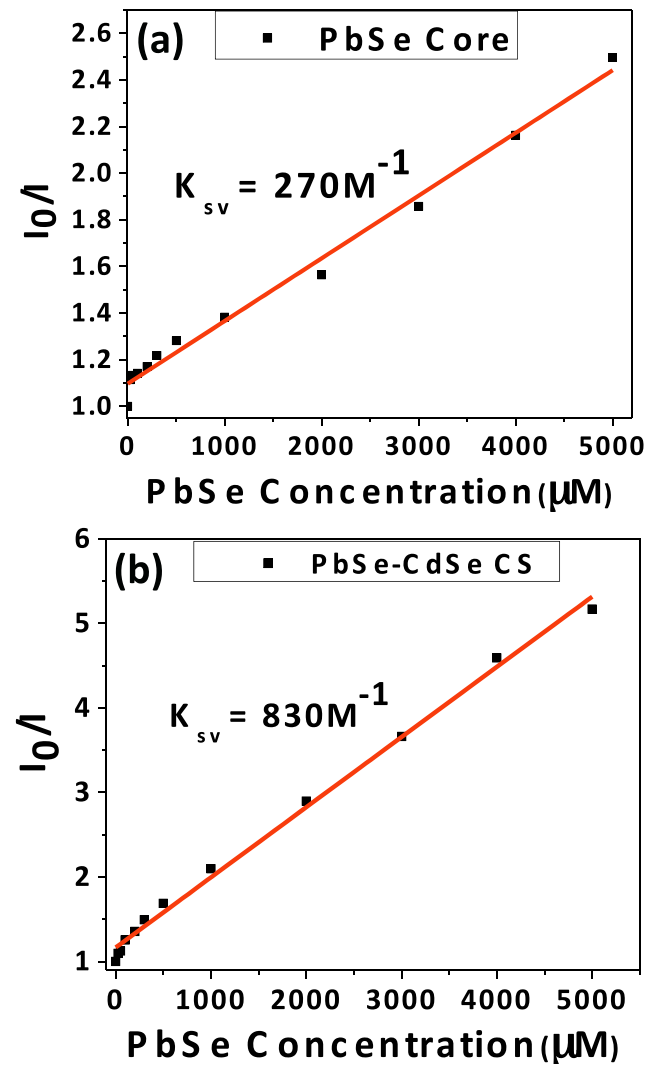

FIG.5. Stern Volmer plot of (a) PbSe core and (b) PbSe-CdSe coreshell (CS) QDs.

The Stern-Volmer quenching constants $\left(\mathrm{K}_{\mathrm{sv}}\right)$ are $270 \mathrm{M}^{-1}$ and $830 \mathrm{M}^{-1}$ for $\mathrm{PbSe} \mathrm{QDs}$ and $\mathrm{PbSe}-\mathrm{CdSe}(\mathrm{CS})$ respectively. It can be noticed that rate of PL quenching $\left(\Delta \mathrm{P}_{\mathrm{L}} / \mathrm{P}_{\mathrm{Li}}\right)$ and sternvolmer quenching constant $\left(\mathrm{K}_{\mathrm{sv}}\right)$ for core-shell nanostructure has been increased significantly as compared to $\mathrm{PbSe}$ core by increasing the concentration of $\mathrm{PbSe}-\mathrm{CdSe} \mathrm{CS}$ inorganic nanostructure in polymer matrix. The increase in rate of PL quenching and stern-volmer quenching constant gives direct evidence that significant charge transfer taking place from polymer to $\mathrm{PbSe}-\mathrm{CdSe} \mathrm{CS}$ QD's. This increased rate of charge transfer is observed due to effective surface passivation of $\mathrm{PbSe}$ core by formation of CdSe shell over it which satisfies the unsaturated bonds present on surface of $\mathrm{PbSe}$ core. The obtained PL results are in the well agreement with other complimentary studies.

Moreover, two photovoltaic devices were also be fabricated on indium tin oxide coated glass substrate used as anode (bottom electrode) in 1:1 ratio of P3HT: PbSe QDs with 
standard device structure ITO/PEDOT: PSS/P3HT: PbSe/Al to watch the IV response and decides the possible application of these optimized nanocomposites in emerging field of Hybrid solar cells (HSCs).

Fig. 6 ( $\mathrm{a} \& \mathrm{~b}$ ) are the IV responses of P3HT: PbSe core and P3HT: PbSe core-shell (CS) based devices respectively. It can be clearly observed from figure, the current density $\left(\mathrm{J}_{\mathrm{sc}}\right)$ and open circuit voltage $\left(\mathrm{V}_{\mathrm{oc}}\right)$ for PbSe QDs and PbSe-CdSe core shell were found to be $2.46 \mu \mathrm{A} / \mathrm{cm}^{2}, 0.25 \mathrm{~V}$ and $5.46 \mu \mathrm{A} / \mathrm{cm}^{2}, 0.43 \mathrm{~V}$ respectively.

The significant improvement achieved in current density and open circuit voltage in case of $\mathrm{PbSe}-\mathrm{CdSe}$ Core shell devices is due to enhanced charge transfer from polymer to core shell QDs than PbSe QDs. This effect is observed due to reduction in unwanted surface defects and traps with better surface passivation by shell formation. It is expected that these defects are hindering the transport of charge carriers towards respective electrodes generated after light absorption in fabricated device.
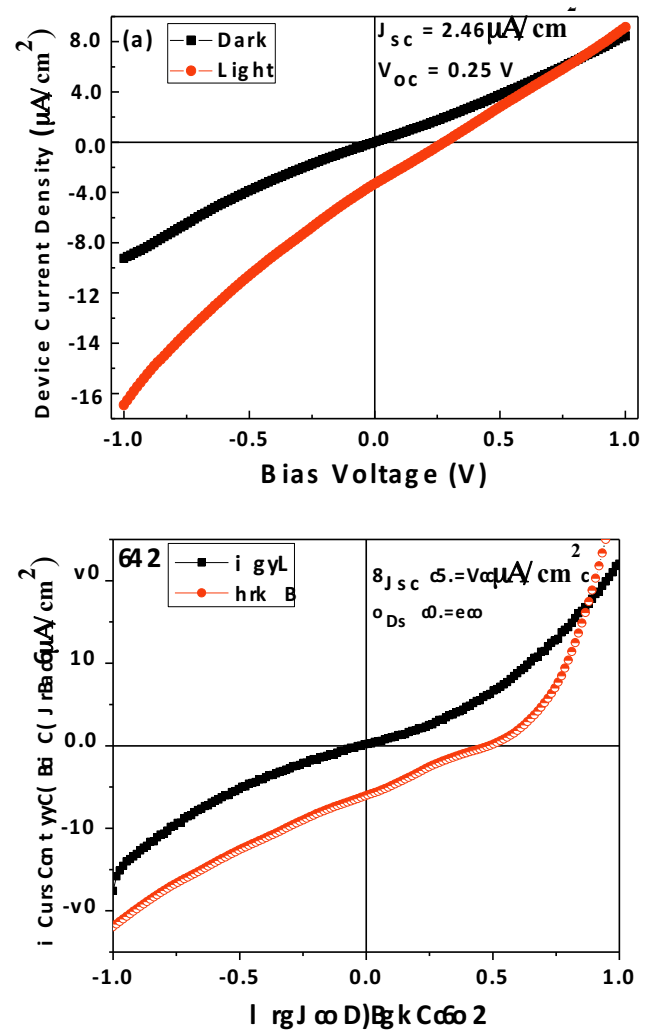

FIG.6. IV response of (a) PbSe Core and (b) PbSe- CdSe core-shell hybrid solar cell.

It could also be perceived from the nature of IV curve that $\mathrm{PbSe} \mathrm{QDs}$ based device having high resistance and low solar effect as compared PbSe-CdSe CS based device. This curve nature might be caused by imperfection of material property or interlayer mismatching during fabrication. An improved IV response for $\mathrm{PbSe}-\mathrm{CdSe} \mathrm{CS}$ based devices was achieved which also support the above findings that CdSe shell formation improved the material property and additionally the device response significantly. The obtained electrical results are in well favor of above results obtained by PL quenching and stern-volmer constant which already proved that charge transfer has enhanced in $\mathrm{PbSe}-\mathrm{CdSe} \mathrm{CS}$ case than PbSe QDs.

Finally, the solar effect was observed for both PbSe-Core and $\mathrm{PbSe}-\mathrm{CdSe}$ core-shell QD's with current in $(\sim \mu \mathrm{A})$ being higher for core-shell than for core. A detailed investigation is underway to improve the current characteristics of these devices.

\section{CONCLUSIONS}

In conclusion, the PbSe Core and $\mathrm{PbSe}-\mathrm{CdSe}$ core-shell QD's are successfully synthesized using oleic acid as a capping ligand by single-pot colloidal route rather than using a conventional double pot synthesis route. The importance and hence novelty of this work being the single-pot synthesis which is simple, convenient, cost-effective and also prevents the oxidation effects of the ambience which significantly enhances the stability of the inorganic PbSe-CdSe core-shell QD's. These synthesized nanostructures having unique optical, structural and electronic properties, are greatly applicable for Hybrid solar cell.

\section{ACKNOWLEDGMENTS}

The authors are grateful to Director, NPL for providing the facilities for the successful completion of this research work. One of the authors Ms. Aarti Mehta is thankful to MNRE for her Senior Research Fellowship.

\section{REFERENCES}

[1] D. Cui, J.Xu, T.Zhu, G. Paradee, S. Ashok and M. Gerhold, Appl. Phys. Lett. 88, 183111 (2006).

[2] L.Levina, V. Sukhovatkin, S. Musikhin, S. Cauchi, R. NNisman and E.H. Sargent, Advanced Material, 17, 1854 (2005).

[3] Sara Bals, M. Casavola, Marjin A. van Huis and Sandra Van Aert, 11, 3420-24 (2011).

[4] Umesh Kumar, Shailesh N. Sharma, Sukhvir Singh, M. Kar, V.N.Singh, B.R.Mehta, Rita Kakkar Material Chem. Phys., 113, 107-114, (2009).

[5] J.Xu, D.Cui, T,Zhu, G.Paradee, Z.Liang, Q.Wang, S.Xu and A.Y. Wang, 17, 5428(2006)

[6] T.T.Tan, S.T. Selvan, L. Zhao, S. Gao and J.Y.Ying, Chem. Mater. 29, 3112 (2007).

[7] Yu Zhang, Quanqin Dai, Xinbi Li, Qingzhou Cui, Zhiyong Gu, Bo Zou and Y wang, nanoscale research letter 5, 1279-83 (2010).

[8] C B Murray, S. Syun, W. Gaschler, H. Doyle, T.A. Betley and C R Kagan, IBM J. Res. Dev. 45 (2001).

[9] Identifying an unknown compound by infrared spectroscopy, prepared by Moses Lee, Furman University.

[10] JianXu, Jian-Ping Ge, and Ya Dong Li, J. Phys. Chem. B, 110, $2497-$ 2501 (2006).

[11] Lakowicz J R 1999 principles of Flourescence Spectroscopy $2^{\text {nd }}$ Edition (New York: Kluwer

[12] KusumKumari, Umesh Kumar, Shailesh N Sharma, Suresh Chand, Rita kakkar, V D vankar and Vikaram Kumar, J Phys D: Appl. Phys. 41 235409(2008). 